\title{
Reducing Teachers' Cognitive Overload with a Recommender System in the Workplace
}

\author{
Arnoldo Rodríguez \\ Universidad de Costa Rica, Turrialba, Costa Rica
}

\begin{abstract}
This paper presents an assistant, ARIALE (Authoring Resources for Implementing Adaptive Learning Environments), that helps decrease the teacher's cognitive load when he has to deal with traditional help techniques, complex tasks or unknown environments in the workplace. The assistant adapts learning support and problemsolving support according to teachers' characteristics, while they are authoring learning sessions to teach decision-making in Network Design. ARIALE applies on-the-job training when a teacher can not spend time learning how to use a tool or how to solve a problem. Our methods for problem-solving support include Bayesian learning to recommend network topologies and our assistant provides adapted help to use a tool rather than teach how to use it.
\end{abstract}

\section{Introduction}

This paper presents an assistant, ARIALE (Authoring Resources for Implementing Adaptive Learning Environments), that involves adapted learning support and problem-solving support for the recommendation of examples that teachers require to author learning sessions for teaching decisionmaking in Network Design.

Our assistant is part of an Adaptive Educational Hypermedia (AEH) intended to support teaching decision-making. ARIALE helps teachers in the workplace, while they are authoring teaching materials, and this helper decreases the user's cognitive load when he has to deal with traditional help techniques or unknown environments.

In the context of learning, cognitive overload is a situation in which the processing demands required by "the learning task may exceed the processing capacity of the cognitive system" of a teacher [10].

We pay attention to aspects intended to reduce cognitive overload problems, such as the excess of help not related to the task on hands, which are often caused by traditional help techniques.
In order to reduce teachers' cognitive overload, problem-solving support helps the user in solving a problem related to Network Design. Instead of helping the user to achieve a goal step-by-step, problem-solving support provides the solution to the problem.

According to the approach of our system for teaching Network Design, an example is a network topology with different number and types of links. Our system includes an implementation of problemsolving support and recommends topologies that the teacher can include in his teaching, when he does not have enough time to create an example from scratch [16].

Learning support is a way to help teachers who do not have enough time to learn the use of a particular tool. Learning support works parallel to the authoring tool, and finds, selects and adapts contextsensitive support for Web-based help, according to a teacher's skills and plans [15]. Then, our system selects the help strategy and techniques to deliver support, the kind of help content to show, and the media to display the content adapted to the teacher's preferences.

This paper also discusses other characteristics of the help provided by ARIALE and we describe the methods that our system uses to adapt help to the teacher's attributes and plan.

\section{Research rationale}

This research addresses two problems that university teachers face when they are authoring teaching materials for their courses:

1. Teachers do not have enough time to create teaching materials.

2.Teachers do not have enough time to learn how to use the tools that could reduce the time required for the creation of materials.

In general, users of software and, particularly, authors of educational material such as teachers face other two difficulties:

1.The production paradox that could be experienced by teachers. The teacher is not familiar with the functionalities of the tool he is going to use, 
he has only a limited potential use of the tool and thus he can not properly use many of the options that the tool provides. As a result, his performance using the tool is low. He can not improve his skills using the tool because he does not have enough time to learn how to use the tool, but, at the same time, he can not learn because he needs the time to perform the tasks that he has to do with the tool [8].

2. A potential cognitive overload could affect teachers using an authoring tool if they split their attention between authoring, finding, and selecting help according to his characteristics to improve their use of the tool. For example, if a teacher is using an authoring tool to create an example, his mind would be involved in three processes at the same time: teaching (which is the main task), manipulating the authoring tool, and dealing with the help features. Doing the tasks simultaneously, he must switch between them. He must carry information from one environment to the other quickly, because he does not have more than 18 seconds to keep information in mind.

In addition, a help system with an index for searching help according to a particular vocabulary or a list of help options can add more headaches to a teacher, because he must know the keywords to find the required help. Thus, teachers authoring learning sessions may well experience a cognitive overload when learning how to use an authoring tool.

General users do not really read the help provided; as they are too eager to start the real work. They only want to know how to do a task even tough they do not know how to access the tools or when to use them, and they are not aware of how to combine, adapt, and modify available tools and functions according to their specific needs. Then, we assume that when an author is creating a learning session with an authoring tool, he faces two main barriers: he requires specific information related to his current task, and he finds it difficult to locate this information.

Sometimes help can be available, but it is not really accessible because users do not know how to access it or access is difficult, or the help is available and accessible, but not adapted to the users' needs. Searching help on Internet usually generates information overload and a loss of time and efforts that become in a hidden cost.

More specifically, two of the problems that teachers may face while authoring a learning session can be 1 . Personalizing help according to each teacher's attributes, and 2. Finding specific help to perform a task. Figure 1. shows an example of a menu he might face to adapt help to use an authoring tool. This menu includes seven different options related to the context, the complexity, language, media, bandwidth, help technique and display mechanism.

\begin{tabular}{|c|c|c|c|}
\hline Context & Activity $=$ & Task $=$ & Step $=$ \\
\hline Complexity & (6) Beginner & C Intermediate & C Advanced \\
\hline Area content & (c) Usage & C Strategy & C Domain \\
\hline Language & c English & C French & CSpanish \\
\hline Media & $\nabla$ Text & V Image & $\ulcorner$ Multimedia \\
\hline Bandwidth & C Low & $6 \mathrm{High}$ & \\
\hline Help strategy & (c Basic & $C$ Guidance & C Assistance \\
\hline
\end{tabular}

\section{Figure1. Possible menu to set up the help for a teacher}

With multiple options, this menu could become more complex if we added the option to navigate between the current and previous help messages displayed. For example, we could add buttons or links to go backward or forward to check these messages.

Finding specific help to perform a task is another problem that teachers may face while authoring a learning session. For example, a teacher can be looking for help related to the task he is performing currently, but the appropriate help can be embedded in a huge document and, then, getting the correct help becomes a time consuming task.

\section{Related work}

Past research has not focused on on-line intelligent and adaptive/hypermedia that helps instructors using Intelligent Tutoring Systems (ITS). However, some authors have mentioned the lack of research on the instructor modelling, the advantages of using information about teachers to improve ITSs [7][3].

WEAR and CACTUS are two of very few systems that specifically address the issue of how to guide teachers using an authoring tool to create an intelligent tutoring system [1][20]. Another important antecedent is Telephone Triage Assistance (TTA), a help system that supports nurses when they are answering by telephone [8]. Moreover, [8] analyses and implements strategies and techniques for on-line help that are very useful to design on-line help and that can be applied to Web-based help systems.

Another important aspect related to examples is that automatic generation of courseware material, particularly examples, is not a common function of ITS and AEH. A system that includes a random problem generator which creates instances of computer networks, by randomly generating values within specified boundaries, is an innovative option to provide support. 
A recurring question in decision-making is how to deal with complex scenarios in which people must make decisions. An attempt to answer this question is Towle's ITS [19] to teach decision-making in complex environments. He proposes simulations of crisis management, using educational multimedia software with a task-based approach and a goalbased scenario. Another antecedent for teaching decision-making skills is "ADELE”, a Web-based agent to support learning that has been applied to teaching medicine [5]. Another previous research study in this area is CACTUS [4], an interactive simulation environment that provides facilities for supporting decision making and whose interface evolves to accommodate and support the varying roles of trainers and trainees.

\section{Solutions}

In this research, teachers' main goal is to create materials for teaching. This is their most important objective, rather than learning to use the authoring tool to be evaluated. Consequently, we have adapted the view of the teacher as learner of new technologies to that of a trainee who requires adaptive help and support to solve problems such as creating case studies. In this scenario, the teacher's learning becomes a by-product of the support that he receives. We see the teacher as a worker who can learn while he is on the job, applying the on-the-jobtraining methods.

\subsection{Pedagogical Model}

A Pedagogical Model models the teaching process, for example, what aspects should be presented to the learner, when to review, when to present a new topic, and which topic to present. Our Pedagogical Model is hybrid because it includes how to support each teacher authoring learning sessions, and this model also contains how to teach a particular subject [16].

Our Pedagogical Model applies an on-the-jobtraining approach to support teachers while they are using the authoring tool for the creation of learning sessions. The on-the-job-training approach is intended to supply support while the teacher is authoring instead of offering training before authoring. Thus, we have considered "on-the-job training” to support the development of on-line help, especially that of Web-based adaptive and intelligent help systems to support authoring tools. We consider that "on-the-job training" is more appropriate for Web-based help than classical methods, which include previous training, pre-test, post-test, and other techniques used to teach and evaluate the learning of a tool. The reason is that teachers are not intended to master specific software or authoring tools to achieve their pedagogical objectives. Then, previous training and tests should be reduced to a minimum.

According to this model, the teacher's learning becomes a by-product of the support that he receives. This is a fundamental characteristic in our research because we do not see the teacher as a typical student. Rather, we see the teacher as a worker who can learn while he is on the job. Seeing the teacher as an author who needs on-the-job training to use an authoring tool is a new view of the teacher's role.

Most people are eager to use any application with no previous test and training and they prefer the "onthe-job training" style focused on real tasks to be performed. This is the reason because we adopted the Electronic Performance Support Systems (EPSS) approach, which provides access to support, tools and resources to perform tasks in a real work environment [8].

EPSS is the definition of a type of on-line help "which provide integrated, on-demand access to information, advice, learning experiences, and tools to enable a high level of job performance with a minimum of support from other people" [8]. Figure 2. shows two different scenarios: a teacher without assistance and a teacher supported by the help system.

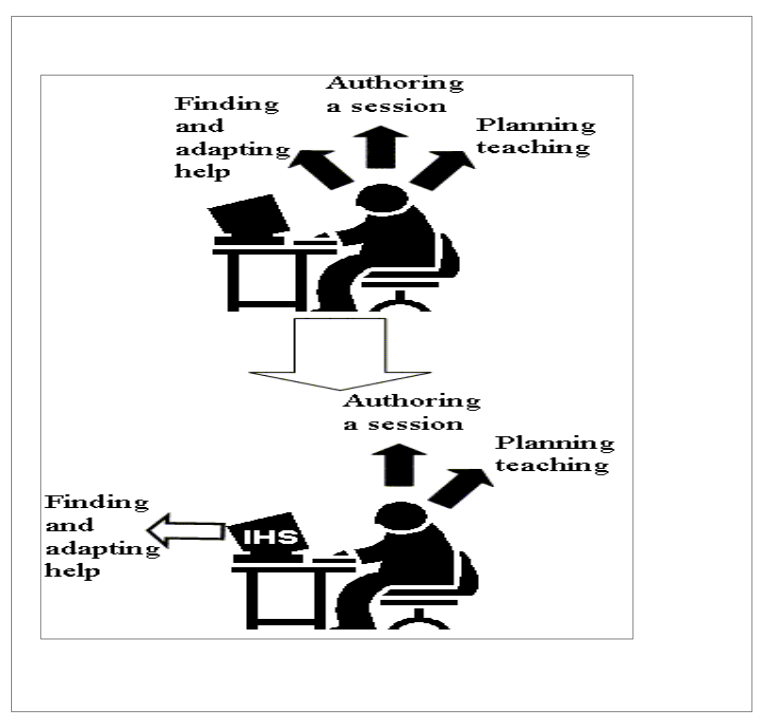

\section{Figure 2. A teacher without support (above) and a helped teacher (below)}

There are three criteria or principles to provide this kind of on-line support.

The first is just-in-time support. With just-in-time support, the system supports the user when he is on the job, at the moment when he needs to develop the competence to perform a task.

The second type is just-enough support. This type means the user is going to receive just enough information to complete the current task. The goal is 
to avoid cognitive overload for the user provoked by information that is not going to be used.

Finally, [8] explains that "most users handle their tasks on hand without necessarily optimizing the solution”. Thus, the idea behind task support systems is that integrating just-in-time support and just-enough support will allow a reduction in the cost of learning a new task. This manner of dealing with help is called continuous performance development. It is assumed that if a user receives just-in-time justenough support, his skills will improve continuously.

According to Cognitive Load Theory (CLT) [18], learning is the product of a process in which the learner uses his limited working memory efficiently. Working memory or short-term memory is the part of the human cognitive process in which cognitive processing are developed. Thus, working memory capacity is a critical factor for learning [9]. This immediate memory limits the amount of information that a person can receive, process and remember around seven (seven plus minus two) chunks of information at a time. Moreover, a human being can deal with a small number of interactive elements, possibly no more than two or three.

A teaching process should free the student's working memory, allowing him to learn chunks of knowledge in an iterative process. After the learner assimilates a part of the knowledge, he frees up his working memory to acquire more complex aspects and so on [9]. Long-term memory stores vast quantities of schemas or patterns that integrate multiple elements of information related to a specific function. A schema organizes information according to the manner in which the person is going to reuse it.

Working memory has a limited capacity to deal with novel information, thus CLT also recommends the use of worked-out examples to reduce the cognitive load that learners may experience while they are learning new concepts.

The option we found to reduce the cognitive load and the production paradox in ARIALE is an Intelligent Help System (IHS) that works parallel to the authoring tool, and finds, selects and adapts context-sensitive help according to a teacher's skills, preferences and current task. IHS provide personalized, dynamic, and contextual support by using artificial intelligence techniques for adaptation. The help features are one way to support the manipulation of the authoring tool and decrease the cognitive load generated by the interaction with this tool.

An intelligent system can perform some tasks by means of artificial intelligence techniques. In general terms, an intelligent system chooses actions based on knowledge to achieve a main objective. This knowledge base can be composed by its experience, data provided by an expert, data acquired from distributed databases, data inferred by itself or data encoded as part of the system structure.

\subsection{The subject}

In this paper, teaching decision-making is seeing as a progressive process to master more complex skills in decision-making [17]. We focus on a particular type of decision-making that is MultiCriteria Decision-Making (MCDM), which concerns multiple conflicting criteria that are the basis for a decision. A criterion is the set of elements (objective, goal and attribute) that the decisionmaker takes into account to evaluate each option. The most important aspect is that two or more attributes can be conflicting; as a result, the solution requires finding a network design with a tradeoff between the conflicting attributes.

In the context of multiple criteria decisionmaking, a problem is a decision to be made, for example, to design a network topology for a bank by taking into account many criteria, such as reliability, the network capacity and number of concentrator. An initial state is a specific configuration of the network topology and a new state is another configuration that results from applying an action, such as adding links to the network to improve its reliability. The new state can be the final design or just an additional step that can be closer to the final decision.

Our approach to develop the student's expertise in decision-making is also supported by CLT [18], which is proposed as a promising tool for developing expertise [13]. In order for students to develop expertise in decision-making, CLT proposes providing problems, with an appropriate difficulty level, in a repetitive process that includes feedback, the opportunity to correct errors and, gradually, problems with an increasing level of difficulty.

The starting point in CLT is cognitive overload, a situation that a user may experience when the processing demands required by a task exceed his processing capacity [10]. For example, while a worker is trying to calculate the average price of some products, at the same time he has to learn how to use a spreadsheet application (software) to calculate the average. Doing the two tasks simultaneously, he must switch between the spreadsheet and the documentation that explains how to use the software to compute the average. In addition, his main goal is to compute the average, not learning to use the software.

According to CLT, learning is the product of a process in which the learner uses his limited working memory efficiently. Working memory or short-term memory is the part of the human cognitive process "in which all conscious cognitive processing occurs". This immediate memory limits the amount of information that a person can receive, process and remember around seven (seven plus minus two) 
chunks of information at a time. Moreover, a human being can deal with a small number of interactive elements, "possibly no more than two or three" [11]. This means that a teaching process should free the student's working memory, allowing him to learn chunks of knowledge in an iterative process. After the learner assimilates a part of the knowledge, he frees up his working memory to acquire more complex aspects and so on. As a result, step by step and chunk by chunk, the learner acquires a big chunk of knowledge, which is located in the long-term memory. Long-term memory stores vast quantities of schemas or patterns that integrate multiple elements of information related to a specific function. A schema organizes information according to the manner in which the person is going to reuse it [2].

CLT in decision-making follows the same strategy used by long-term memory: a learner acquires a simple pattern used for making decisions and, iteratively, he learns more complex patterns until achieving a high level of expertise. Finally, he can retrieve a complex high-level schema from the long-term memory by putting lower level schemas in the working memory to process situations that require expert skills. For example, a person can learn how to make decisions about designing a network with a minimum degree of network reliability; after that he can learn more about the location of concentrators to support an acceptable degree of network reliability; in a third activity, this person can learn how the assignation of capacity to different links increase or decrease the network cost. Once he has accumulated knowledge about network reliability, concentrator location and capacity allocation, he will apply these three aspects together in the design of a network, a more complex activity that integrates the previous three.

Moreover, working memory has a limited capacity to deal with novel information, thus CLT also recommends the use of worked-out examples to reduce the cognitive load that learners may experience while they are learning new concepts.

"Learning by doing" with worked-out examples, the learner can develop the skills to recognize patterns in the provided examples. In this way, the student develops the expertise to retrieve schemas from his long-term memory to compare them with the examples and identify differences and similarities.

Payr defines five phases that a learner can pass through as he progresses: novice, beginner, competence, proficiency and expertise [12]. In each phase, the learner performs some activities intended to achieve some skills: receive and remember; imitate and apply; select and decide; understand and design; recognize and master. Table 1 . shows the five phases and their corresponding activities.
Table 1. Phases of the learning process, according to [12].

\begin{tabular}{|l|l|l|}
\hline & Phase & Activity \\
\hline & & \\
\hline 1 & NOVICE & Receive, remember \\
\hline 2 & BEGINNER & Imitate, apply \\
\hline 3 & COMPETENCE & Select, decide \\
\hline 4 & PRO-EFICIENCY & Understand, design \\
\hline 5 & EXPERTISE & Recognize, master \\
\hline
\end{tabular}

In addition, this view defines the corresponding skills that a learner can develop as he passes through the different phases from novice to expertise: skills to deal with free-context situations, skills to act in context-sensitive conditions, skills for problem solving, skills to recognize patterns, and skills for managing complex situations. Thus, this approach is fundamental not only for teaching decision-making skills to students gradually, but also for adapting support to teachers as they progress and accumulate experience in using an authoring tool, for example.

\subsection{Recommendations}

While a teacher is authoring a learning session, he has to include examples that students will analyze. The creation of examples is a time-consuming task for teachers; as a consequence, ARIALE is an assistant that simplifies the situation for the teacher by means of two main functions:

1. Generating, testing and recommending examples and learning session to teachers (problemsolving support).

2. Offering adaptive context-sensitive and Webbased help about how to use the authoring tool (learning support).

Problem-solving support can be implemented by means of a recommender system. To recommend topologies, our system accesses a case base that stores topologies and selects a series of examples matching the teacher's teaching style (teaching style is the class of topologies that a teacher prefers) [16]. If a teacher has not authored any sessions or does not want old sessions created by other teachers, our system generates examples according to the criteria defined by the designer. ARIALE generates, tests and recommends additional examples by using a probabilistic method, which assigns at random the links between the nodes of a topology. ARIALE also verifies that any generated example fits in the class of examples used by the teacher. If the system generates an example that is out of the scope that the teacher prefers, the example is aborted and a new one is generated. We use Bayesian learning to test if a generated example matches the teacher's teaching style; if the example matches his style, ARIALE recommends the topology. 
Let us study an instance of testing an example (a classification process), given a training set with two classes of networks as shown in Table 2.

\section{Table 2. A set of classes to classify} examples (training set).

\begin{tabular}{|c|l|l|c|}
\hline Example & Attribute 1 & Attribute 2 & Class \\
\hline & Reliability & Cost & \\
\hline 1 & High & Costly & A \\
\hline 2 & Very high & Costly & A \\
\hline 3 & Very high & Costly & A \\
\hline 4 & High & Very costly & B \\
\hline 5 & High & Very costly & B \\
\hline
\end{tabular}

We need to test a network $E$ ' with a cost attribute which is "Very costly" and a reliability attribute which is "Very High". In this case, the example $E$ ' must fit in class b to be accepted. There is not any "Very high" reliability in class B and there is not any "Very costly" cost in class A. In which class must the new example be classified?

To verify if the example fits in Class $B$ and is accepted or if it must be rejected, we first have to calculate the probabilities:

\section{$P($ Very costly $\mid A), P($ VeryHigh $\mid A)$}

$P($ Very costly $\mid B), P($ VeryHigh $\mid B)$

We apply equation (1) to calculate these probabilities:

$$
P\left(a_{i} v_{j}\right)=\frac{n_{c}+m p}{n+m}
$$

where:

- $\quad a_{i}$ is the value of each attribute $a_{i}$ tested for each class $v_{j}$.

- $\quad n$ is the total number of instances in each class $v_{j}$. Class A has three instances and class B has two.

- $\quad n_{c}$ is the number of instances with attribute $a_{i}$ and class $v_{j}$. Class A has two instances of "Very High" and Class B has none.

- $\quad p$ is a priori estimate for $P\left(a_{i} \mid v_{j}\right)$. According to [6] $p$ means the probability of $a_{i}$ having one of its possible values; in this case $P$ is $1 / 2$ because we assume that the probabilities of all attributes are equiprobable (equally likely to be true).

- $\quad m$ is a constant used to avoid the possible consequences if $n_{c}=0$ (in this case the calculation would be zero). We use $\mathrm{m}=2$ as a constant because we have two attributes (Reliability and Cost) in our training set.

We calculate the probability of the example to be classified in A and the probability to be classified in $B$. Then we apply the equation (1) $m$ times for each class. Table 3 displays the values for $n, n_{c}, p$ and $m$.
Table 3. Values to calculate where the example can be classified.

\begin{tabular}{|c|c|c|}
\hline \multicolumn{2}{|l|}{ Class A } & Class B \\
\hline $\begin{array}{l}\text { Very High } \\
n=3 \\
p=.5\end{array}$ & $\begin{aligned} n c & =2 \\
m & =2\end{aligned}$ & $\begin{array}{l}\text { Very High } \\
n=2 \\
p=.5\end{array}$ \\
\hline $\begin{array}{l}\text { Very Costly } \\
n=3 \\
p=.5\end{array}$ & $\begin{aligned} n c & =0 \\
m & =2\end{aligned}$ & $\begin{array}{ll}\text { Very Costly } & \\
n=2 & n c=2 \\
p=.5 & m=2 \\
\end{array}$ \\
\hline
\end{tabular}

Using the values in Table 3, it is possible to get the final results using equation (2):

$$
v\left(E^{\prime}\right)=\underset{v_{j} \in V}{\operatorname{argmax}} P\left(v_{j}\right) \prod_{i} P\left(a_{i} \mid v_{j}\right)
$$

where:

1. $V$ is the set of classes $v_{j}$.

2. $P\left(v_{j}\right)$ is the overall probability of a class. This means the frequency of each class in the training set. The probability for Class $\mathrm{A}$ is $3 / 5$ and that for Class B is $2 / 5$.

In our example we adjust the expression to:

$$
v(E)=\underset{v_{j} \in|A, B|}{\operatorname{argmax}} P\left(v_{j}\right) \prod_{i} P\left(a_{i} \mid v_{j}\right)
$$

where $\{A, B\}$ is the set of classes available.

Then, multiplying the probabilities for each attribute, we get the probabilities for each class:

$$
P(\mathrm{~A})=0.6 * 0.2=0.12
$$

$P(B)=0.25 * 0.75=0.1875$

Now, each result has to be multiplied by the corresponding $P\left(v_{j}\right)$ :

$$
\begin{aligned}
& P(\mathrm{~A})=0.12 * 0.6=0.072 \\
& P(\mathrm{~B})=0.1875 * 0.4=0.075
\end{aligned}
$$

This calculation can also be performed as follows:

$$
\mathrm{P}(\mathrm{A}) * \mathrm{P}(\text { VeryCostly } / \mathrm{A}) * \mathrm{P}(\text { VeryHigh } / \mathrm{A})=0.6 * 0.6 *
$$
$0.2=0.072$

$\mathrm{P}(\mathrm{B}) * \mathrm{P}($ VeryCostly $/ \mathrm{B}) * \mathrm{P}($ VeryHigh $/ \mathrm{B})=0.4 * 0.25 *$ $0.75=0.075$

Finally, a network with a cost "Very costly" and reliability "High" must be classified in Class B, because $0.075>0.072$. Then, the network passed the test.

Learning in naïve Bayesian classifier derives from updating the probabilities based on the frequencies in the training data. In addition, there is nothing to search in a tree or a database; thus, the classification of a new instance involves only computing the maximum class posterior probability [6]. Furthermore, a Bayesian classifier does not compare an example that is yet to be classified with those 
already classified. Instead of this, a Bayesian classifier can keep a summarized image of the frequency with which different characteristics of an example appear in the database.

\subsection{Adaptive interface}

A goal of the IHS is to adapt the interface contents and their presentation to the user according to his plan, skills and preferences. Changing the interface of the authoring tool and the embedded context-sensitive help requires to choosing different resources. ARIALE takes into account data about the teacher's skills, checks the plan to follow, and decides the type of learning support or problemsolving support that the teacher requires. The learning support helps the teacher to perform some tasks. Our system includes three help strategies that are Basic Help, Guidance, and Assistance [15].

Basic help is a strategy oriented to provide justenough contextual help or problem-solving support. Just-enough contextual help means the system displays only short messages with the most relevant information related to a specific aspect of the current Web page. Problem-solving support provides a complete solution for a problem; in our case problem-solving support is the recommendation of a worked-out example to be reused. Guidance is intended to supply short information about how to perform a specific task. These strategies can explain a concept either in brief or in detail, depending on the teacher's skills (beginner, intermediate or advanced).

Accessing help is a type of navigation through the help contents. When a teacher clicks links to view help or when the system provides active help, this interaction allows the teacher to traverse the nodes of the help hypermedia. The authoring tool and the help system act as parallel layers.

In many applications, a teacher must switch from one window to another window or frame to check the help information. This type of switching from one window to another requires the teacher to remember (to keep in his short-term memory) help information from a help window in order to apply it in the authoring tool. Sometimes the help window can be confused with other windows and the switching process becomes more disruptive. This is a cognitive overload that needs to be eliminated or at least reduced. In order to offer less disruptive help, our system has a standard layout for all the Web pages in the site. We decided that

-the left area of the screen is used to display the menu for navigation,

-the authoring area uses the middle area of the screen,

-the right area of the screen is used to display help information.
Figure 3. shows the standard layout of the user interface for the Web site.

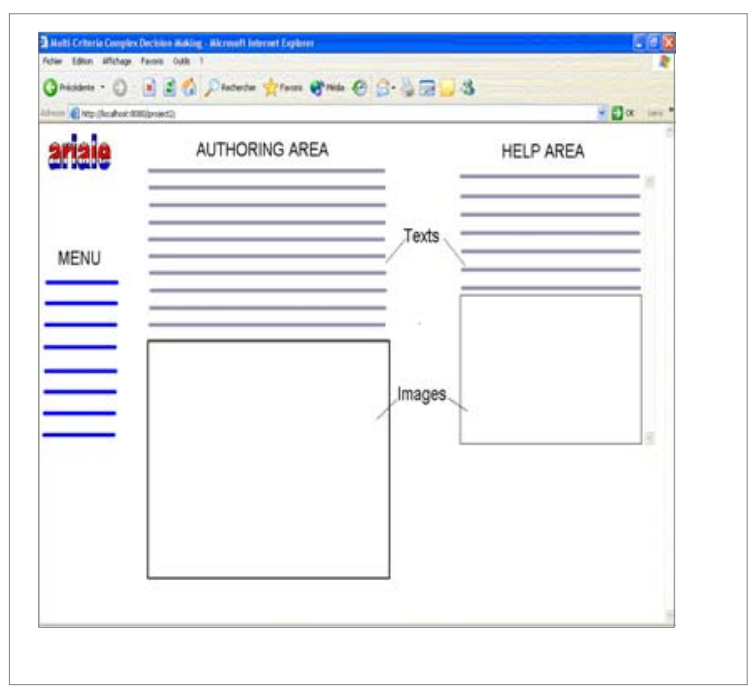

\section{Figure 3. The standard layout of the user interface}

Another aspect related to the interaction of teachers with the help system is the help is embedded. Thus, the help appears within the same window, rather than in a separate help window. In the case of problem-solving support, when an example is recommended, it is loaded in the Webpage.

\section{Structure}

ARIALE has a client-server architecture with a three-tier structure that includes most processes working on the server-side, the database on the server-side, and a client-side [17].

On the server side, the system includes components such as Curriculum, Teacher Model, Pedagogical Model, Sessions Base, Resources, a Session Manager, and a Helper. On the client side, an adaptive interface interacts with the user.

The system has some standard templates for the different steps of the tasks, and, when a teacher finishes the actions to be done on a page, a new record is stored in the database. Some instances of templates are associated with specific pages or steps of a task in a session. A program on the server side generates the code of the template.

\section{Evaluation}

We applied the accuracy criterion to check if the system recommends good examples of topologies. In our validation, we not only tested the accuracy of our Bayesian classifier but also compared it to a kNN (k=1 Nearest Neighbour) method [14][15]. The 
accuracy of our recommendation method was validated by the following two ways:

- 10-fold cross-validation.

- Comparison of the performance of the Bayesian classifier with the other classifier.

We ran 10-fold cross-validation to validate our classifiers, and the accuracy of our Bayesian classifier based on the measures of performance is $94 \%$. This means that this classifier has 6\% error according to the test developed with a training dataset of 200 topologies (20 topologies for testing and 180 for training for each test). This classifier works with six classes of topologies.

According to a 10-fold cross-validation, the accuracy of a k-NN ( $\mathrm{k}=1$ Nearest Neighbour) classifier based on the measures of performance is $96 \%$ for the classification of network topologies in ARIALE.

Although the result of testing the accuracy of kNN classifier (96\%) is better than the result obtained by the Naïve Bayes classifier (94\%), a Bayesian classifier is more flexible and easy to adapt to different domains and number of attributes.

The evaluation of the acceptance of the help approach applied in ARIALE includes the analysis of automatic generation and recommendation of examples (problem-solving support), and our learning support approach. For this study, we selected 27 teachers that work in the Universidad de Costa Rica (a public university in San José, Costa Rica) [14].

More that the half of teachers does not use teaching material that can be generated automatically, but $45 \%$ (12) of teachers think, at least partially, that the automatic generation and recommendation of examples reduces the time required to produce teaching materials.

\section{Conclusions}

This paper focuses on the manipulation of the help to free the teacher's mental capacity and allow him to pay better attention to the translation of his plans in the authoring tool. The help features are one way to support the manipulation of the authoring tool and decrease the cognitive load generated by the interaction with this tool. Another important aspect to distinguish our option is the integration of the generation, testing and recommendation of examples as part of the intelligent help system in ARIALE. Worked-out examples are stored as cases that ARIALE manipulates applying artificial intelligence techniques.

In addition, we consider that our learning support approach that involves on-the-job training and learning by doing as part of an electronic performance support system is a good alternative to formal training when there is no enough time available.

\section{Acknowledgements}

This research was supported by the University of Costa Rica, the Consejo Nacional para Investigaciones Científicas y Tecnológicas, and the Ministerio de Ciencia y Tecnología de Costa Rica. This work is also supported partially by the National Sciences and Engineering Research Council of Canada (NSERC), the Fonds Québécois de la Recherche sur la Nature et les Technologies (FCAR), and Valorisation Recherche Québec (VRQ) of the governments of Canada and Quebec.

\section{References}

[1] F. García, "CACTUS: Automated tutorial course generation for software applications", Proceedings of the 5th International Conference on Intelligent User Interfaces, USA, 2000, pp. 113-120.

[2] P. Gerjets, and K. Scheiter, "Goal configurations and processing strategies as moderators between instructional design and cognitive load: Evidence from hypertext-based instruction”, Educational Psychologist, Vol. 38, Great Britain, 2003, pp. 33-41.

[3] S. Graf, Kinshuk, C. Ives, “A flexible mechanism for providing adaptivity based on learning styles in learning management systems", 10 IEEE International Conference on Advanced Learning Technologies, 2010, pp. 30-34.

[4] R. Hartley, "An interactive computer-based simulation environment for supporting and developing complex decision-making skills”. Int. J. Cont. Engineering Education and Lifelong Learning, Vol 13, Nos. 3/4, 2003, pp. 212-231.

[5] W. Johnson, E. Shaw, A. Marshall, C. LaBore, "Evolution of user interaction: the case of agent Adele", Proceedings of the $8^{\text {th }}$ International Conference on Intelligent user interfaces, USA, 2003, pp. 93-100.

[6] F. Keller, "Naive Bayes Classifiers", Connectionist and Statistical Language Processing, Course at Universit"at des Saarlandes, Germany, 2002: http://homepages.inf.ed.ac.uk/keller/teaching/connectionis m/lecture10_4up.pdf. Accessed January 2004.

[7] Kinshuk, "State-of-the-art in Adaptive Learning: Keynote Address Pedagogies \& Communication”, Proceedings of the International Conference on Open \& Online Learning (ICOOL 2003), University of Mauritius, 2003:

http://icool.uom.ac.mu/2003/papers/file/keynote/kinshuk.zi p Accessed March 2004.

[8] M. Leung, “Towards a generic approach to providing proactive task support", Ph.D. Dissertation, University of Waterloo, Canada, 2001. 
[9] T. Lin and Kinshuk, "Adaptive techniques for supporting working memory capacity", Proceedings of the International Conference on Computers in Education (ICCE2004), Australia, 2004, pp. 7871-792.

[10] R. Mayer and R., Moreno, "Nine ways to reduce cognitive load in multimedia learning”, Educational Psychologist, Vol. 38, Great Britain, 2003, pp. 43-52.

[11] F. Pass, A. Renkl, J. Sweller, "Cognitive Load Theory and Instructional Design: Recent Developments", Educational Psychologist, Vol. 38, No. 1, Great Britain, 2003, pp. 1-4.

[12] S. Payr, “The Virtual University's faculty: An overview of educational agents", Applied artificial intelligence, Vol. 17, Germany, 2003, pp. 1-19.

[13] R. Rickers, P. Gerven, H. Schmid, "Cognitive Load Theory as a tool for Expertise Development”, Instructional Science, Vol. 32, Netherlands, 2004, pp. 173-182.

[14] A. Rodríguez, "Evaluación de un tipo de ayuda en línea destinada a profesores que deseen crear sitios educativos en Internet”, Intersedes, Vol. VII, Número 11, Costa Rica, 2007, pp. 99-117.

[15] A. Rodríguez, "Generation, Testing and Recommendation of Teaching Materials Using Classification Learning”, International Journal of Advanced Media and Communication (IJAMC), Vol. 2, Número. 3, Switzerland, 2008, pp. 308 - 324.

[16] A. Rodríguez, “Teacher Model supporting an educational adaptive hypermedia for an implementer teacher", Proceedings of the Canada International Conference on Education (CICE-2011), Canada, 2011, pp. 531-536.

[17] A. Rodríguez, "Integrating methods to build a recommender system with adapted help", Proceedings of EDULEARN11 Conference, Spain, 2011, pp. 5336-5344.

[18] J. Sweller, “Cognitive load during problem solving: Effects on learning”, Cognitive Science, Vol. 12, 1988, pp. 257-285.

[19] B. Towle, "Authoring tools for building how-to simulations", Ph.D. dissertation, Northwestern University, USA. 2001.

[20] M. Virvou and M. Moundridou, “Adding an instructor modelling component to the architecture of ITS authoring tools", International Journal of Artificial Intelligence in Education, Vol. 12, 2002, pp. 185-211. 